\title{
An Okinawan-based Nordic diet improves glucose and lipid metabolism in health and type 2 diabetes, in alignment with changes in the endocrine profile, whereas zonulin levels are elevated (Review)
}

\author{
BODIL OHLSSON ${ }^{1,2}$ \\ ${ }^{1}$ Department of Clinical Sciences, Lund University, Lund S-221 00; ${ }^{2}$ Department of Internal Medicine, \\ Skåne University Hospital, Malmö S-205 02, Sweden
}

Received October 21, 2018; Accepted January 28, 2019

DOI: $10.3892 /$ etm.2019.7303

\begin{abstract}
The Okinawan-based Nordic diet has been developed to improve glucose metabolism. The aim of the present study was to summarize all anthropometric, subjective, and biochemical findings obtained following two different studies investigating this diet. The diet was administered i) as a single breakfast to healthy volunteers and ii) as a 12-week dietary intervention to patients with type 2 diabetes. The degree of satiety, sweet cravings, gastrointestinal symptoms, and health-related quality of life were estimated. Weight and blood pressures of participants were measured, and analyses including circulating levels of inflammatory and metabolic biomarkers, hormones, and short-chain fatty acids (SCFA), and microbial diversity and amount of Enterobacteriaceae in feces, were performed. A single breakfast of the diet increased satiety $(\mathrm{P}<0.001)$, improved glucose homeostasis $(\mathrm{P}<0.001)$, and lowered levels of glucose-dependent insulinotropic
\end{abstract}

Correspondence to: Professor Bodil Ohlsson, Department of Internal Medicine, Skåne University Hospital, 15 Jan Waldenström Street, Malmö S-205 02, Sweden

E-mail: bodil.ohlsson@med.lu.se

Abbreviations: AUC, area under the curve; BMI, body mass index; CCK, cholecystokinin; Hs-CRP, hypersensitive C-reactive protein; ELISA, enzyme linked immunosorbent assay; GI, glycemic index; GLC, gas-liquid chromatography; GLP-1, glucagon-like peptide-1; GIP, glucose-dependent insulinotropic polypeptide; HbA1c, glycated hemoglobin A1c; HOMA2-IR, homeostasis model assessment for insulin resistance; HRQoL, health-related quality of life; INF- $\gamma$, interferon gamma; IL, interleukin; PAI-1, plasminogen activator inhibitor-1; PYY, polypeptide YY; qPCR, quantitative polymerase chain reaction; SCFA, short-chain fatty acids; T-RFLP, terminal restriction fragment length polymorphism; TNF- $\alpha$, tumor necrosis factor alpha; VAS, visual analogue scale

Key words: calprotectin, glucose homeostasis, hormones, interleukins, lipid homeostasis, Okinawan-based Nordic diet, type 2 diabetes, weight, zonulin polypeptide $(\mathrm{GIP})(\mathrm{P}=0.002)$, compared with a standard breakfast. A 12 -week intervention in type 2 diabetes increased satiety and decreased sweet cravings, at the same time as health-related quality of life and gastrointestinal symptoms were improved. There were reductions in body mass index $(\mathrm{P}<0.001)$, waist circumference $(\mathrm{P}<0.001)$, and levels of glucose $(\mathrm{P}<0.001)$, cholesterol $(\mathrm{P}<0.001)$, and triglycerides $(\mathrm{P}=0.009)$, in alignment with the endocrine profile. These improvements were maintained at follow-up 16 weeks later, along with lower levels of ghrelin $(\mathrm{P}=0.012)$, polypeptide $\mathrm{YY}(\mathrm{P}=0.002)$, and visfatin $(\mathrm{P}=0.021)$, compared with the parameters recorded at the study start. Levels of haptoglobin, interleukin-18 and thrombocytes were lowered, whereas some other inflammatory biomarkers were unaffected and zonulin levels elevated. Gut microbiota and SCFAs levels were mainly unaffected. The mechanisms governing the anthropometric and metabolic improvements appear to be mediated through alterations in the endocrine profile, yet not in the gut microbiota.

\section{Contents}

1. Introduction

2. Experimental procedures

3. Subjective assessment

4. Anthropometry

5. Metabolic effects

6. Endocrine effects

7. Inflammatory parameters

8. Gut microbiota and metabolomics

9. Mechanisms behind the improved anthropometry and metabolism

10. Conclusion

\section{Introduction}

Diabetes and obesity are diseases with increasing prevalence, mainly depending on an unhealthy diet and less physical activity (1), with major influences on circulating levels of biomarkers. Lifestyle factors are difficult to study since 
many various factors are involved and may be confounders, rather than causalities. Despite extensive research, there is no consensus of which diet is best to maintain health. Modern Western diet is anticipated to induce both a low-grade inflammation and an increased intestinal permeability $(2,3)$, reflected by elevated circulating zonulin levels (4). Epidemiological studies strongly suggest that the Mediterranean diet with a high amount of fruits and vegetables reduces the inflammation $(5,6)$. The research studies are inconclusive concerning the amount and type of fat which is most optimal for the metabolism (7).

Since adipose tissue constitutes a strong source for endocrine and inflammatory mediators (2), it is customary to know whether the diet, or the weight reduction after introduction of a healthier diet, contribute to the improved metabolism, endocrine profile, and inflammatory state. The adipokines leptin, resistin, and visfatin play a central role in the control of energy metabolism, regulation of glucose and lipid metabolism, and insulin sensitivity (8), and are supposed to be involved in the development of auto-immunity, diabetes, inflammation, metabolic syndromes, and obesity (9). Elevated levels of plasminogen activator inhibitor-1 (PAI-1) form a link between obesity, insulin resistance, and the risk of cardiovascular events (10). Cortisol is assumed to be involved in the development of the metabolic syndrome and type 2 diabetes (11). Both luminal carbohydrates and fat induce postprandial secretion of the incretins glucagon-like peptide-1 (GLP-1) and glucose-dependent insulinotropic polypeptide (GIP) $(12,13)$, which also play roles in the regulation of gastrointestinal motility, obesity development, and secretion of luminal digestive enzymes (13-16). Cholecystokinin (CCK) and polypeptide YY (PYY) are released in response to fat and protein, and constitute important hormones in the regulation of appetite, body weight, gastrointestinal motility $(17,18)$. Ghrelin concentrations are suppressed in response to meal intake (19), whereas basal levels are increased after weight reduction (20).

The composition of gut microbiota is mostly determined by the diet (21). A high microbial diversity with many different species seems to be of importance for health (22). Our Western lifestyle with high consumption of carbohydrates, saturated fat, and processed food promotes a lower diversity. Disturbances in the microbiota, i.e., dysbiosis, seem to be of importance for the development of our most common diseases, e.g., allergy, hypertension, malignancy, obesity, and type 2 diabetes $(23,24)$.

The Okinawan diet is a fiber-rich diet based on the eating habits of the people of the Ryukyu Islands, where average life expectancy is very high with five times as many Okinawans who live to be 100 years old as their compatriots elsewhere in Japan (25). The diet shares many similarities with the Mediterranean diet (6). Based on the traditional Okinawan food (25), a modified diet has been developed to suit tastes and food components suitable for the Nordic population. The food is based on traditional Nordic raw food, e.g., berries, fruits, nuts, leguminous, root crops, vegetables, whole-grains, and fatty fish, with moderately low carbohydrate energy content with higher contents of fiber, fat, and protein (26).

The aim of the present review about the Okinawan-based Nordic diet was to summarize the most important findings after a single breakfast in healthy volunteers and after a 12-week dietary intervention in type 2 diabetes.

\section{Experimental procedures}

To examine the effects evoked by the Okinawan-based Nordic diet, two separate studies were performed at the Skåne University Hospital, Malmö, Sweden (Table I).

First, 20 healthy volunteers (12 women), mean age $46.0 \pm 14.5$ years, body mass index (BMI) $24.6 \pm 2.7 \mathrm{~kg} / \mathrm{m}^{2}$, had to randomly ingest a breakfast on two separate days, after an over-night fast. Study participants were recruited from hospital staff and their family members. In one morning, an ordinary Swedish breakfast was ingested, and in another morning, an Okinawan-based Nordic breakfast was ingested. Blood samples were drawn before breakfast and at intervals until at $180 \mathrm{~min}$ afterwards, at the same time as they scored their degree of satiety $(27,28)$.

Second, 45 patients diagnosed with type 2 diabetes were randomly selected from a health care center in the south of Sweden. After consideration of inclusion and exclusion criteria of other concomitant severe diseases, 30 patients (17 women) with type 2 diabetes, mean age $57.5 \pm 8.2$ years, mean disease duration $10.4 \pm 7.6$ years, BMI $29.9 \pm 4.1 \mathrm{~kg} / \mathrm{m}^{2}$, had the Okinawan-based Nordic diet delivered for 12 weeks. Thus, all the participants ingested the same diet, except the breakfast which the participants had to prepare themselves. The participants were allowed to eat three meals a day including breakfast, lunch, and dinner, and two snacks between meals consisting of a variety of fruits, berries, and seeds. The meal composition is close to a diet with moderately low carbohydrate energy content with higher contents of fiber, fat, and protein (26). It consists of ordinary food, but with minimal industrial processing. Organic food items are preferred whenever possible. The content of high glycemic index (GI) food is reduced radically (e.g., white flour and sugar) along with cakes, dairy products, red meat, and processed meat (Table II). At the same time, the gluten content is decreased since wheat meal is reduced and only two slices of bread is given each day. The meals start with ingestion of green salad or raw vegetables: $100 \mathrm{~g}$ at breakfast and $150 \mathrm{~g}$ at lunch and dinner, respectively. The diet supplies all essential nutrients to recommended levels or above, including a mean calorie intake around $1900 \mathrm{kcal} / \mathrm{day}$, which is slightly lower than the traditional Nordic diet (Table III). The diet is rich in omega-3 fats with a high monounsaturated-to-saturated fat ratio (29).

The breakfasts consisted of either porridge or fermented milk, depending on their ordinary habits, in addition to bread. Those who did not get any fermented milk product to breakfast ingested some form of fermented milk as a snack. The participants were instructed not to change any other lifestyle habits during the study time. The food was delivered pre-prepared to the participants three times a week, along with recipes for the final meal preparation. The major differences between the diet compared to their ordinary food habits were that protein intake was previously dominated by meat (65.5\% had a few meat intakes a week and $34.5 \%$ had daily meat intake) over fish $(86.1 \%$ had fish intake once a week or less). Furthermore, root and other vegetable intakes were relatively low, with $55.2 \%$ of participants consuming these foods a few times a week or less, $37.9 \%$ once daily and $6.9 \%$ more than once daily (29). The participants were followed for another 16 weeks. The participants were examined at study 
Table I. Characteristics of the two studies.

\begin{tabular}{lll}
\hline Study design & \multicolumn{1}{c}{ Breakfast study } & \multicolumn{1}{c}{ Dietary interventional (O-BN diet) study } \\
\hline $\begin{array}{l}\text { Study population } \\
\text { Type of dietary intervention }\end{array}$ & $\begin{array}{l}\text { Healthy volunteers (n=19) } \\
\text { 2 different breakfasts (one } \\
\text { O-BN-based and one standard) } \\
\text { ingested at two separate time }\end{array}$ & $\begin{array}{l}\text { T2DM patients (n=30 at 12 weeks and n=23 at 28 weeks) } \\
\text { 12 week long dietary intervention with the O-BN diet }\end{array}$ \\
& $\begin{array}{l}\text { points } \\
\text { Th (3.5 h per breakfast) }\end{array}$ & 28 weeks \\
Total participant study time & $\begin{array}{l}\text { Biomarkers and satiety scores } \\
\text { (pre- and until 180 min }\end{array}$ & $\begin{array}{l}\text { Anthropometric measurement } \\
\text { Blood and feces sampling (baseline and week 2,6, 12, 28) } \\
\text { (time-points for measurement) }\end{array}$ \\
& postprandial) & Study questionnaires (baseline and week 12, 28) \\
\hline
\end{tabular}

n, number; O-BN diet, Okinawan-based Nordic diet; T2DM, type 2 diabetes.

Table II. Food items in the Okinawan-based Nordic diet.

\begin{tabular}{ll}
\hline Food groups & Food types \\
\hline
\end{tabular}

Beverages/liquids

Dairy products

Egg

Fat and oil

Fruits and berries

Legumes

Nuts and seeds

Rice and cereals

Root vegetables

Vegetables

Fish and seafood

Meat products and poultry

Spices

Sweets and deserts
Recommended free amount of tap water or mineral water with the meals. Light products to be avoided. Tea, filtered coffee, or instant coffee. Maximal intake of alcoholic beverages was set to $30 \mathrm{~g}$ ethanol/week.

Low-fat drinking milk (maximum 1.5\% fat), filmjölk (a Swedish fermented product similar to yogurt) and low-fat yogurt (1.5\% fat), hard cheese (17\% fat), parmesan cheese, cottage cheese, and quark. Whipped cream (36\% fat) and soy cream used for cooking, and Turkish yogurt (10\% fat).

Eggs were used for cooking and as snacks.

Vegetable oil including rapeseed oil, sesame oil, and olive oil for cooking and dressing. Vegetable fat spread (60\% fat) for bread.

Apple, orange, pear, strawberry, blueberry, lingonberry, dried apricots, and prunes. Coco nut milk was used for cooking.

Fresh; green peas, sweet peas, and soybean.

Dried; white and black beans, kidney beans, and lentils.

Walnuts, almonds, and cashews. Sunflower seeds, pumpkin seeds, linseed, and sesame seed, as part of snacks served with fruits and berries.

Whole-grain rice and black Thai rice. A maximum of half a dl of uncooked rice per serving. Rolled oats, rye flakes, whole-grain whole-kernel rye, oat, barley, and wheat. Bread rich in whole-grain, a maximum of two slices per day. Whole-grain pasta, bean paste, millet, oat bran, quinoa, and rye flour.

Hot or cold. Red or yellow carrots as snacks or incorporated in the dish. Parsnips, parsley root, rutabaga, celery root, beetroot, artichoke, or sweet potatoes as part of the dishes.

Shredded in potatoes burger, fried raw.

Onion, leek, garlic, cabbage, cauliflower, broccoli, squash, eggplant, fennel, spinach, various types of salad, tomato, pepper, cucumber, mushroom, and asparagus.

Salmon, codfish, plaice, mackerel, herring, and prawns.

Chicken, rooster, turkey, game meat (hart and deer), and ground beef.

Own preference with restrictions on salt.

Homemade dark chocolate $(>70 \%)$, bean truffles, coconut flakes, ginger, and prune cake. No refined sugar or sweetener was used, but instead natural sweet foods like prunes, pears, and occasionally honey. start and after 2, 6, 12, and 28 weeks when anthropometry and compliance were assessed along with collection of blood and feces samples. Questionnaires about medical history, nutrition, socioeconomic factors, health-related quality of life (HRQoL) measured by RAND 36 (30), gastrointestinal symptoms measured by the visual analog scale for irritable bowel 
Table III. Nutrition composition and daily mean intake of energy of the modified Okinawan-based Nordic diet, compared with Nordic Nutrition Recommendations (NNR) 2012.

\begin{tabular}{llccc}
\hline Nutritional value & Unit & Calculated value & E\% & Recommended (NNR 2012) \\
\hline Total energy & $\mathrm{kcal}$ & 1866.0 & & \\
Energy (excluding beverages) & $\mathrm{kcal}$ & 1629.0 & 42 & $45-60 \mathrm{E} \%$ \\
Carbohydrates & $\mathrm{g}$ & 168.4 & 6 & $<10 \mathrm{E} \%$ \\
Sucrose & $\mathrm{g}$ & 23.5 & 4 & $25-35 \mathrm{~g}$ \\
Dietary fiber & $\mathrm{g}$ & 35.9 & 35 & $25-40 \mathrm{E} \%$ \\
Fat & $\mathrm{g}$ & 63.9 & 10 & $<10 \mathrm{E} \%$ \\
Saturated fatty acids & $\mathrm{g}$ & 18.7 & 8 & $5-10 \mathrm{E} \%$ \\
Polyunsaturated fatty acids & $\mathrm{g}$ & 14.9 & 10 & $10-20 \mathrm{E} \%$ \\
Monounsaturated fatty acids & $\mathrm{g}$ & $\mathrm{g}$ & 23 & $10-20 \mathrm{E} \%$ \\
Protein & $\mathrm{g}$ & 95.0 & \\
\hline
\end{tabular}

The daily average calorie content of the meals are given in kcal and the energy percentage (E\%) is given for nutrients, based on the intake of a normal week. Recommendations for a traditional diet according to the Nordic Nutrition Recommendations are shown for comparison (26). Recommendations for total daily energy intake are not given since they are individual and differ between subjects.

syndrome (VAS-IBS), and satiety and sweet craving measured by two separate visual analog scales (VAS), were completed at baseline, and after 12 and 28 weeks (31). At all the time points, the participants met a nutritionist and completed a food diary during the intervention. The nutritionist also followed the patients weekly by mail or telephone.

The Department of Clinical Chemistry analyzed cortisol, C-peptide, and insulin in serum; blood counts and glycated hemoglobin A1c (HbA1c) in blood; and electrolytes, glucose, inflammatory parameters, iron status, kidney, liver, and thyroid function tests, and lipid status in plasma. Homeostasis model assessment for insulin resistance (HOMA2-IR) was calculated using the HOMA2 calculator version 2.2.3 (32). Luminex analyses were performed in plasma samples to measure the levels (pg/ml) of ghrelin, glucagon, GLP-1, GIP, leptin, PAI-1, resistin, and visfatin $(27,33)$, and ELISA was used to analyze plasma levels of CCK and PYY (33). Calprotectin and zonulin in serum and feces were analyzed by ELISA (34). The concentrations of the pro-inflammatory cytokines tumor necrosis factor alpha (TNF- $\alpha$ ), interferon gamma (IFN- $\gamma$ ), interleukin 1 alpha (IL-1 $\alpha$ ), IL-1 $\beta$, IL-12 p70, IL-17A and IL-18, and the anti-inflammatory cytokines IL-2, IL-4 and IL-10 were quantified in un-diluted plasma using a custom premixed human magnetic 10-plex (35). Gut microbiota was analyzed in feces by terminal restriction fragment length polymorphism (T-RFLP) to determine microbial diversity. Thresholds for internal standard and terminal restriction fragments (T-RFs) were set to 7 and 20 fluorescence units, respectively. Microbial diversity was estimated by calculation of richness (number of T-RFs) and Shannon-Wiener and Simpson's diversity indices, with the exception that T-RFs within 40-580 base pairs were included in the T-RFLP profile analysis and calculation. The diversity indices take into accountability both richness and evenness when considering the relative abundance of bacterial groups. Both indices are commonly used to assess microbial diversity. Samples under the detection limit in the qPCR runs were set to the highest possible detection limit for statistical analysis. Quantitative PCR was used to estimate the amount of
Enterobacteriaceae (29). Short-chain fatty acids (SCFAs) were determined in serum by gas-liquid chromatography (GLC) (29). For further information on methods, see original papers.

Paired t-test was used for calculations of differences in the breakfast study. Linear mixed effect models were used to analyze differences in continuous variables and Fisher's exact test was used for dichotomous variables in the 12-week dietary intervention. Spearman's correlation test was used for absolute and partial correlations (controlling for subject) in both studies. $\mathrm{P}<0.05$ was considered to indicate a statistically significant difference.

\section{Subjective assessment}

An increased satiety was experienced after a single breakfast of the Okinawan-based Nordic diet compared to a standard breakfast $(\mathrm{P}<0.001)(27)$. After a 12-week dietary intervention in type 2 diabetes, the patients scored their satiety equal (27\%) or increased (60\%) compared with baseline (31), although hormones normally inducing satiety, such as CCK, glucagon, GLP-1, insulin, leptin, and PYY, were reduced during the intervention. The levels of the orexigenic hormone ghrelin were decreased at 16 weeks after the intervention had ceased (Table IV). Satiety was not correlated with any hormones, except an inverse correlation with GIP at week 28 (rs $=-0.450$, $\mathrm{P}=0.041)(31)$.

Furthermore, the participants scored their sweet craving as less during and after the dietary intervention in comparison to baseline $(\mathrm{P}=0.002)$, without correlations with any metabolic or endocrine changes at week 12 (31). However, after another 16 weeks, VAS scores of sweet craving correlated with leptin levels ( $\mathrm{rs}=0.458, \mathrm{P}=0.037$ ), weight ( $\mathrm{rs}=0.426, \mathrm{P}=0.048)$, $\mathrm{BMI}$ $(\mathrm{rs}=0.651, \mathrm{P}=0.001)$, and waist circumference ( $\mathrm{rs}=0.565$, $\mathrm{P}=0.006$ ). No other anthropometric, hormonal, or metabolic parameters correlated with the overall sensations of satiety and sweet craving measured in VAS scales (31).

Symptoms of diarrhea and bloating declined, and psychological well-being was improved, during the intervention. At week 16 after completion, the symptoms abdominal pain and 
Table IV. Hormonal concentrations after an intervention with an Okinawan-based Nordic diet.

$\begin{array}{ll}\frac{95 \% \mathrm{CI}}{\text { Mean }} & \\ \text { Lower Upper P-value }\end{array}$

$\mathrm{CCK}(\mathrm{pg} / \mathrm{ml}$; plasma)

Baseline

Week 12

Week 28

Cortisol (nmol/l; serum)

Baseline
Week 12
Week 28
C-peptide (nmol/l; serum)

Baseline

Week 12

Week 28

Ghrelin (pg/ml; plasma)

Baseline

Week 12

Week 28

Glucagon (pg/ml; plasma)

Baseline

Week 12

Week 28

GLP-1 (pg/ml; plasma)

Baseline

Week 12

Week 28

GIP (pg/ml; plasma)

Baseline
Week 12
Week 28
Insulin (mIU/1; serum)
Baseline
Week 12
Week 28

Leptin (pg/ml; plasma)

Baseline

Week 12

Week 28

PAI-1 (pg/ml; plasma)

Baseline

Week 12

Week 28

PYY (pg/ml; plasma)

Baseline

Week 12

Week 28

Resistin (pg/ml; plasma)

Baseline

Week 12

Week 28

0.022

0.015

0.249

$348 \quad 315 \quad 382$

0.005

$20 \quad 14 \quad 26$

0.267

$\begin{array}{lll}367 & 337 & 397\end{array}$

$325 \quad 290 \quad 360$

$\begin{array}{llll}0.99 & 0.82 & 1.2 & \\ 0.88 & 0.70 & 1.0 & 0.022 \\ 0.88 & 0.68 & 1.1 & 0.131\end{array}$

$\begin{array}{cccc}852 & 608 & 1,094 & \\ 798 & 555 & 1,041 & 0.219 \\ 728 & 481 & 975 & 0.012\end{array}$

$\begin{array}{llll}1.9 & 1.3 & 2.6 & \\ 1.3 & 0.6 & 1.9 & 0.003 \\ 1.7 & 1.1 & 2.4 & 0.426\end{array}$

$\begin{array}{llll}2.7 & 1.8 & 3.5 & \\ 1.8 & 0.9 & 2.7 & 0.013 \\ 2.4 & 1.4 & 3.3 & 0.477\end{array}$

$<0.001$

$\begin{array}{rrrr}55 & 34 & 76 & <0.001 \\ 75 & 52 & 98 & 0.692\end{array}$

$\begin{array}{lll}16 & 13 & 18\end{array}$

$\begin{array}{lll}12 & 9 & 15\end{array}$

0.004

$\begin{array}{llll}13 & 10 & 16 & 0.089\end{array}$

$9,404 \quad 587 \quad 1,294$

$\begin{array}{llll}5,850 & 199 & 971 & <0.001\end{array}$

$\begin{array}{llll}8,553 & 491 & 1,219 & 0.171\end{array}$

$72,434 \quad 58,869 \quad 85,998$

$\begin{array}{llll}66,413 & 52,402 & 80,425 & 0.082\end{array}$

$\begin{array}{llll}71,810 & 56,705 & 86,915 & 0.889\end{array}$

$\begin{array}{rrrr}1,937 & 1,802 & 2,073 & \\ 1,510 & 1,360 & 1,660 & <0.001 \\ 1,673 & 1,502 & 1,844 & 0.002\end{array}$

Table IV. Continued.

\begin{tabular}{|c|c|c|c|c|}
\hline & \multirow[b]{2}{*}{ Mean } & \multicolumn{2}{|c|}{$95 \% \mathrm{CI}$} & \multirow[b]{2}{*}{ P-value } \\
\hline & & Lower & Upper & \\
\hline \multicolumn{5}{|c|}{ Visfatin (pg/ml; plasma) } \\
\hline Baseline & 998 & 732 & 1,264 & \\
\hline Week 12 & 1,115 & 816 & 1,415 & 0.235 \\
\hline Week 28 & 812 & 535 & 1,089 & 0.021 \\
\hline \multicolumn{5}{|c|}{$\begin{array}{l}\text { Values are presented as absolute mean values and } 95 \% \text { confidence } \\
\text { interval }(\mathrm{CI}) \text { with lower and upper limits at inclusion (baseline) } \\
(n=30), 12 \text { weeks after diet intervention }(\mathrm{n}=30) \text {, and } 16 \text { weeks after } \\
\text { the end of diet intervention (week } 28)(n=23) \text {. CCK=cholecystokinin, } \\
\text { GLP-1=glucagon-like peptide- } 1, \mathrm{GIP}=\text { glucose-dependent insuli- } \\
\text { notropic polypeptide, PAI- } 1=\text { plasminogen activator inhibitor- } 1 \text {, } \\
\text { PYY=polypeptide YY. Linear mixed model. Comparisons were made } \\
\text { between baseline and week } 12 \text { and week } 28 \text {. P }<0.05 \text { was considered } \\
\text { to indicate a statistically significant difference. }\end{array}$} \\
\hline
\end{tabular}

nausea were significantly reduced compared with baseline, and the mean scores for diarrhea and bloating were further improved. No correlations were seen between symptoms and improvements of anthropometric, hormonal, or metabolic parameters (31).

The health concepts physical functioning, general health, vitality, social functioning, and mental health were improved throughout the study (30). There were strong correlations between HRQoL and gastrointestinal symptoms, and the improvement in $\mathrm{HRQ} \mathrm{OL}$ preceded the improvements of gastrointestinal symptoms (31).

\section{Anthropometry}

During the interventional period, there were progressive and significant mean reductions already after 2 weeks in body weight $(-3.03 \mathrm{~kg}, \mathrm{P}<0.001)$, BMI $(-1.00 \mathrm{~kg} / \mathrm{m} 2, \mathrm{P}<0.001)$, and waist circumference $(-3.19 \mathrm{~cm}, \mathrm{P}<0.001)$, which continued to 12 weeks afterwards. Despite some increases, these parameters remained significantly lowered compared with baseline until 16 weeks after completed intervention. A significant reduction in the systolic and diastolic blood pressures could be seen during the dietary intervention, and the diastolic blood pressure remained lowered after 28 weeks compared with baseline $(-1.75 \mathrm{mmHg}, \mathrm{P}=0.001)$. The progressive mean reductions in the heart and respiratory rates could be seen already 2 weeks after the dietary introduction, persisting throughout the complete study period (Table V) (30).

\section{Metabolic effects}

The postprandial elevation of plasma glucose, calculated as differences of maximal levels, was reduced in healthy volunteers after the Okinawan-based Nordic breakfast compared with the traditional breakfast $(-0.88 \mathrm{mmol} / 1, \mathrm{P}<0.001)$, along with a reduced secretion of C-peptide $(-366 \mathrm{pg} / \mathrm{ml}$, $\mathrm{P}=0.011)$, insulin $(-379 \mathrm{pg} / \mathrm{ml}, \mathrm{P}=0.007)$, and GIP $(-192 \mathrm{pg} / \mathrm{ml}$, 
Table V. Anthropometric and metabolic parameters in type 2 diabetes before and after a 12-week Okinawan-based Nordic diet intervention.

\begin{tabular}{|c|c|c|c|c|}
\hline \multirow[b]{2}{*}{ Variable } & \multirow{2}{*}{$\begin{array}{l}\text { Mean } \\
\text { value }\end{array}$} & \multicolumn{2}{|c|}{$95 \% \mathrm{CI}$} & \multirow[b]{2}{*}{ P-value } \\
\hline & & Lower & Upper & \\
\hline \multicolumn{5}{|c|}{ Weight (kg) } \\
\hline Baseline & 89.8 & 84.5 & 95.1 & \\
\hline Week 12 & 83.6 & 78.1 & 89.0 & $<0.001$ \\
\hline Week 28 & 85.4 & 79.7 & 91.1 & $<0.001$ \\
\hline \multicolumn{5}{|c|}{$\mathrm{BMI}\left(\mathrm{kg} / \mathrm{m}^{2}\right)$} \\
\hline Baseline & 29.9 & 28.4 & 31.3 & \\
\hline Week 12 & 27.8 & 26.3 & 29.4 & $<0.001$ \\
\hline Week 28 & 28.4 & 26.8 & 30.0 & $<0.001$ \\
\hline \multicolumn{5}{|c|}{ Waist circumference $(\mathrm{cm})$} \\
\hline Baseline & 107 & 103 & 111 & \\
\hline Week 12 & 100 & 96.1 & 104 & $<0.001$ \\
\hline Week 28 & 102 & 97.6 & 106 & $<0.001$ \\
\hline \multicolumn{5}{|c|}{$\begin{array}{l}\text { Systolic blood pressure } \\
(\mathrm{mmHg})\end{array}$} \\
\hline Baseline & 140 & 135 & 146 & \\
\hline Week 12 & 131 & 124 & 137 & $<0.001$ \\
\hline Week 28 & 140 & 134 & 146 & 0.796 \\
\hline \multicolumn{5}{|c|}{$\begin{array}{l}\text { Diastolic blood pressure } \\
(\mathrm{mmHg})\end{array}$} \\
\hline Baseline & 82.3 & 78.7 & 85.9 & \\
\hline Week 12 & 74.9 & 71.0 & 78.7 & $<0.001$ \\
\hline Week 28 & 78.7 & 74.8 & 82.7 & 0.001 \\
\hline \multicolumn{5}{|c|}{ Fasting glucose (mmol/l) } \\
\hline Baseline & 9.71 & 8.54 & 10.9 & \\
\hline Week 12 & 7.91 & 6.55 & 9.27 & $<0.001$ \\
\hline Week 28 & 9.28 & 7.71 & 10.8 & 0.466 \\
\hline \multicolumn{5}{|c|}{ HbA1c (mmol/mol) } \\
\hline Baseline & 61.6 & 56.4 & 66.7 & \\
\hline Week 12 & 49.2 & 44.0 & 54.4 & $<0.001$ \\
\hline Week 28 & 54.4 & 48.8 & 59.9 & 0.002 \\
\hline \multicolumn{5}{|c|}{ HOMA2-IR (U) } \\
\hline Baseline & 3.00 & 2.50 & 3.51 & \\
\hline Week 2 & 2.53 & 1.96 & 3.09 & 0.012 \\
\hline Week 12 & 2.37 & 1.82 & 2.91 & $<0.001$ \\
\hline Week 28 & 2.61 & 2.05 & 3.16 & 0.025 \\
\hline \multicolumn{5}{|c|}{ Triglycerides (nmol/l) } \\
\hline Baseline & 1.79 & 1.41 & 2.16 & \\
\hline Week 12 & 1.49 & 1.09 & 1.89 & 0.009 \\
\hline Week 28 & 1.96 & 1.46 & 2.46 & 0.367 \\
\hline \multicolumn{5}{|c|}{ Cholesterol (mmol/l) } \\
\hline Baseline & 4.65 & 4.36 & 4.95 & \\
\hline Week 12 & 4.22 & 3.87 & 4.57 & 0.001 \\
\hline Week 28 & 4.71 & 4.37 & 5.05 & 0.636 \\
\hline \multicolumn{5}{|c|}{$\mathrm{HDL}(\mathrm{mmol} / \mathrm{l})$} \\
\hline Baseline & 1.22 & 1.10 & 1.35 & \\
\hline Week 12 & 1.19 & 1.05 & 1.32 & 0.267 \\
\hline Week 28 & 1.34 & 1.20 & 1.47 & 0.003 \\
\hline
\end{tabular}

Table V. Continued.

\begin{tabular}{llllll}
\hline & & \multicolumn{2}{c}{$95 \% \mathrm{CI}$} & \\
\cline { 3 - 4 } Variable & $\begin{array}{l}\text { Mean } \\
\text { value }\end{array}$ & Lower & Upper & P-value \\
\hline LDL (mmol/l) & & & & \\
Baseline & 2.92 & 2.62 & 3.22 & \\
Week 12 & 2.68 & 2.33 & 3.03 & 0.041 \\
Week 28 & 2.82 & 2.49 & 3.16 & 0.356 \\
\hline
\end{tabular}

The mean values and mean changes and $95 \%$ confidence interval (CI) with lower and upper limits are presented for anthropometric parameters and for fasting blood levels of glycated hemoglobin A1c (HbA1c) and fasting plasma levels of glucose, triglycerides, cholesterol, high-density lipoprotein (HDL), and low-density lipoprotein (LDL) at inclusion (baseline) $(\mathrm{n}=30), 12$ weeks after diet intervention $(n=30)$ and 16 weeks after the end of diet intervention (week 28) $(n=23)$. Insulin resistance was measured by HOMA2-IR (ref no 32). Linear mixed model. Comparisons were made between baseline and week 12 and week 28 . P-value $<0.05$ was considered to indicate a statistically significant difference.

$\mathrm{P}=0.002)$ (28). The postprandial levels of insulin correlated strongest with levels of triglycerides and GIP (Table VI). There were no correlations between glucose and insulin levels and between glucose and GIP levels after the breakfasts (27). During the intervention, a significant reduction was observed already after 2 weeks in the mean fasting blood levels of glucose $(-1.80 \mathrm{mmol} / 1, \mathrm{P}<0.001)$ and serum levels of proinsulin $(-10.15 \mathrm{pmol} / \mathrm{l}, \mathrm{P}=0.005)$ and insulin $(-2.81 \mathrm{mIU} / \mathrm{l}, \mathrm{P}=0.011)$, accompanied with reduced levels of $\mathrm{C}$-peptide and $\mathrm{HbAlc}$ after 12 weeks. At follow up, glucose and C-peptide levels had returned to basal levels, whereas HbAlc and insulin levels were still lowered (Tables IV and V) (30).

None of the participants increased their anti-diabetes treatment during the study. However, in 15 cases (54\% of the subjects on medical treatment), the anti-diabetes medication had to be gradually reduced during the study period, and in two cases, including one on insulin treatment, the medication was cancelled. Out of the remaining 12 diabetes patients treated with insulin, three got their insulin therapy cancelled and another eight had their insulin doses reduced with $27.6 \pm 16.4 \%$ in average (30).

Although the Okinawan-based Nordic breakfast had a fat content of $20.3 \mathrm{~g}$ compared with $14.1 \mathrm{~g}$ in the control breakfast, the postprandial delta area under the curve (dAUC) of triglycerides was more increased after the latter $(\mathrm{P}<0.001)$ than after the test meal $(\mathrm{P}=0.011)$, with no difference between breakfasts $(\mathrm{P}=0.579)$ (28). Plasma levels of fasting cholesterol (-0.44 mmol/1, $\mathrm{P}=0.001)$, low-density lipoprotein (LDL) $(-0.24 \mathrm{mmol} / \mathrm{l}, \mathrm{P}=0.041)$, and triglycerides $(-0.30 \mathrm{mmol} / \mathrm{l}$, $\mathrm{P}=0.009)$ were lowered during the 12 -week intervention, whereas levels of high-density lipoprotein (HDL) was higher at follow-up ( $0.11 \mathrm{mmol} / 1, \mathrm{P}=0.003)$ (30). There were strong correlations between lipid values and glucose and HbAlc values in type 2 diabetes during the intervention (Table VII).

Blood counts, iron status tests, kidney function tests, liver function tests (except for gamma glutamyl transpeptidase 
Table VI. Significant correlations in healthy volunteers after breakfast intake of the Okinawan-based Nordic diet.

\begin{tabular}{|c|c|c|c|c|}
\hline & \multicolumn{2}{|c|}{ Okinawan-based Nordic breakfast } & \multicolumn{2}{|c|}{ Ordinary Swedish breakfast } \\
\hline & Correlation coefficient & $\mathrm{P}$-value & Correlation coefficient & $\mathrm{P}$-value \\
\hline \multicolumn{5}{|l|}{ Insulin } \\
\hline Triglycerides 45 & 0.585 & 0.008 & 0.146 & 0.562 \\
\hline Triglycerides 60 & 0.653 & 0.002 & 0.391 & 0.109 \\
\hline Triglycerides 90 & 0.434 & 0.063 & 0.559 & 0.016 \\
\hline Triglycerides 120 & 0.532 & 0.023 & 0.838 & $<0.001$ \\
\hline Triglycerides 180 & 0.468 & 0.043 & 0.588 & 0.010 \\
\hline \multicolumn{5}{|l|}{ Insulin } \\
\hline GIP 0 & 0.460 & 0.041 & 0.640 & 0.002 \\
\hline GIP 10 & 0.720 & $<0.001$ & 0.633 & 0.004 \\
\hline GIP 20 & 0.728 & $<0.001$ & 0.819 & $<0.001$ \\
\hline GIP 30 & 0.766 & $<0.001$ & 0.804 & $<0.001$ \\
\hline GIP 45 & 0.579 & 0.007 & 0.460 & 0.048 \\
\hline GIP 60 & 0.528 & 0.017 & 0.565 & 0.012 \\
\hline GIP 90 & 0.501 & 0.024 & 0.516 & 0.024 \\
\hline GIP 120 & 0.608 & 0.004 & 0.631 & 0.003 \\
\hline GIP 180 & 0.604 & 0.005 & 0.438 & 0.054 \\
\hline
\end{tabular}

GIP, Glucose-dependent insulinotropic polypeptide. Plasma samples were taken at start and after 10, 20, 30, 45, 60, 90, 120, and 180 mins. Spearman's correlation test. $\mathrm{P}<0.05$ was considered statistically significant, and only correlations with significant changes are shown.

Table VII. Partial correlations in diabetes between fasting levels of glucose and lipid metabolism.

\begin{tabular}{lcc}
\hline & Correlation coefficient & P-value \\
\hline Cholesterol & 0.303 & 0.006 \\
Glucose & 0.346 & 0.001 \\
HbA1c & & \\
Triglycerides & 0.680 & $<0.001$ \\
Glucose & 0.528 & $<0.001$ \\
HbA1c & & 0.087 \\
LDL & 0.190 & 0.093 \\
Glucose & 0.187 & \\
HbA1c & & 0.003 \\
HDL & -0.327 & 0.004 \\
Glucose & -0.314 & \\
HbA1c & & \\
\hline
\end{tabular}

HbA1c, Hemoglobin A1c; HDL, High-density lipoprotein; LDL, Low-density lipoprotein. Partial correlations, with the three observational time points baseline, week 12 and week 28 from each individual, by Spearman's correlation test. P Spearman's correlation test. $\mathrm{P}<0.01$ was considered to indicate a statistically significant difference.

(GGT); $0.23 \mu \mathrm{kat} / \mathrm{l} ; \mathrm{P}=0.016)$, thyroid-stimulating hormone (TSH), cobalamin, folate, albumin/creatinine ratio (ACR) and the apolipoprotein B/apolipoprotein A1 ratio were unaffected during and after the study period (30).

\section{Endocrine effects}

Total AUC (tAUC) of C-peptide (-29, P=0.033), GIP (-32, $\mathrm{P}=0.004)$, and insulin $(-15, \mathrm{P}=0.006)$ in serum or plasma showed attenuated postprandial values after an Okinawan-based Nordic breakfast intake compared with the standard breakfast in healthy volunteers, whereas postprandial tAUC of ghrelin $(-26, \mathrm{P}=0.230)$, glucagon $(-0.6, \mathrm{P}=0.480)$, GLP-1 (-1.6, $\mathrm{P}=0.280)$, leptin (44, $\mathrm{P}=0.320)$, PAI-1 (38, $\mathrm{P}=0.250)$, resistin $(6, \mathrm{P}=0.730)$, and visfatin $(-16, \mathrm{P}=0.260)$ were equal between the two meals (27). The intervention reduced levels of CCK, C-peptide, glucagon, GLP-1, GIP, insulin, leptin, PAI-1, and PYY. After 28 weeks, levels of PYY were still decreased in addition to ghrelin and visfatin (Table IV) (33).

At baseline, levels of C-peptide correlated with glucose $(\mathrm{rs}=0.376, \mathrm{P}=0.041)$ and triglycerides $(\mathrm{rs}=0.399, \mathrm{P}=0.029)$, and the correlation with triglycerides persisted at week 12 ( $\mathrm{rs}=0.388, \mathrm{P}=0.037)$ and week $28(\mathrm{rs}=0.485, \mathrm{P}=0.022)$. PAI-1 levels correlated with cholesterol ( $\mathrm{rs}=0.451, \mathrm{P}=0.035)$ and triglycerides ( $\mathrm{rs}=0.458, \mathrm{P}=0.032$ ) at week 28 . Leptin levels correlated with BMI at baseline ( $\mathrm{rs}=0.430, \mathrm{P}=0.019)$, week 12 ( $\mathrm{rs}=0.478, \mathrm{P}=0.012)$, and week 28 ( $\mathrm{rs}=0.589, \mathrm{P}=0.004)$, whereas insulin levels only correlated with BMI at week 12 ( $\mathrm{rs}=0.419$, $\mathrm{P}=0.029$ ). There were strong correlations between levels of GIP and C-peptide after 12 weeks ( $\mathrm{rs}=0.593, \mathrm{P}=0.001)$ and GIP and insulin after another 16 weeks ( $\mathrm{rs}=0.532, \mathrm{P}=0.011)$.

Cortisol levels continued to decline after both breakfasts in healthy volunteers due to the diurnal rhythm (28) and during the dietary intervention, irrespective of any measured anthropometric and metabolic parameters or symptoms (Table IV) (30). 
The vast majority of subjects continued with this breakfast after the intervention and were still eating this at follow-up. When calculating correlations between food components and hormones, there was a negative correlation at week 12 between resistin and the total amount of carbohydrates ( $\mathrm{rs}=-0.581, \mathrm{P}=0.005)$ and fiber $(\mathrm{rs}=-0.616, \mathrm{P}=0.002)$. This was still observed at follow-up ( $\mathrm{rs}=-0.481, \mathrm{P}=0.027$ and $\mathrm{rs}=-0.432$, $\mathrm{P}=0.050$, respectively). Similar inverse correlations were observed between visfatin and energy percentage of carbohydrates at week 12 (rs=-0.433, P 0.057) and at follow-up ( $\mathrm{rs}=-0.465, \mathrm{P}=0.034)$.

\section{Inflammatory parameters}

Hypersensitive C-reactive protein (hs-CRP) levels were elevated in several of the diabetes patients at study start (mean value: $3.7 \mathrm{mg} / \mathrm{l}$; $95 \%$ confidence interval (CI); reference value: 1.8-5.6 mg/l). The values of albumin, hs-CRP, and leukocytes were unaffected throughout the study, whereas the levels of haptoglobin in plasma and thrombocytes in blood were lowered at follow-up compared with prior study start. Neither the food components nor the hormone levels did correlate with the inflammatory parameters such as albumin, hs-CRP, haptoglobin, leukocytes, or thrombocytes (34). A single meal did not affect cytokine levels in plasma, whereas plasma concentrations of IL-18 were decreased after a 12-week intervention, which correlated with levels of glucose, HbAIc, and lipids both at baseline, and after 12 and 28 weeks (35), but not with any food items (data not shown).

A single breakfast intake did not affect the plasma secretion of calprotectin or zonulin. There were no correlations between the two biomarkers in fast ( $\mathrm{rs}=-0.730, \mathrm{P}=0.841)$ or at $3 \mathrm{~h}$ postprandial ( $\mathrm{rs}=-0.115, \mathrm{P}=0.751)(28)$. Calprotectin levels in serum were within normal ranges but tended to increase during the dietary intervention $(370.8 \mathrm{ng} / \mathrm{ml}, \mathrm{P}=0.074)$, and returned to basal values at week 28 . Feces calprotectin values were higher than the normal reference level $(<50 \mathrm{mg} / \mathrm{kg})$ at study start and was decreased non-significantly throughout the study. Calprotectin levels were not influenced by age, gender, weight, or glucose levels, but correlated with CRP, leukocytes, PAI, resistin, and visfatin after the 12-week dietary intervention (34).

Serum levels of zonulin were elevated already at baseline, and was further elevated during the dietary intervention (12.9 ng/ml, $\mathrm{P}=0.019)$, which persisted during the follow-up (15.2 ng/ml, $\mathrm{P}=0.014)$. On the contrary, the feces levels were only higher than expected after 12 weeks of dietary intervention $(42.9 \mathrm{ng} / \mathrm{ml}, \mathrm{P}<0.001)$. Serum zonulin correlated positively with weight, BMI, and waist circumference at baseline, and with levels of glucose, triglycerides, cholesterol, and LDL, but not with any inflammatory biomarkers, after the dietary intervention (34).

No correlations could be found between food composition and calprotectin or zonulin in serum or feces, or between calprotectin and zonulin, at study start. However, correlations were seen of these two parameters both between serum and feces levels, respectively, after dietary introduction and at follow-up (34). At week 12, energy percentage of protein in breakfast was strongly correlated with calprotectin in serum ( $r s=0.499, \mathrm{P}=0.008)$, which was further strengthened at follow-up ( $\mathrm{rs}=0.687, \mathrm{P}<0.001)$. Zonulin in serum had a tendency to correlate with energy percentage of protein and correlated inversely with energy percentage of carbohydrates at follow-up (34).

\section{Gut microbiota and metabolomics}

The dietary intervention did neither affect microbial diversity nor the amount of fecal Enterobacteriaceae. The only changes in SCFA concentrations, i.e., acetic acid, butyric acid, propionic acid, isobutyric acid, and isovaleric acid, were a decreased level of isovaleric acid after 12 weeks $(\mathrm{P}=0.018)$ and of butyric acid after 28 weeks $(\mathrm{P}=0.007)$. In addition, the ratios acetic acid/propionic acid and acetic acid/propionic acid and butyric acid were also decreased ( $\mathrm{P}=0.050$ vs. $\mathrm{P}=0.003$ ) (29).

\section{Mechanisms behind the improved anthropometry and metabolism}

The main dietary effects found by an Okinawan-based Nordic diet were increased satiety and lower glucose levels, accompanied by lower levels of GIP, C-peptide, and insulin. These changes are supposed to be the primary events in the introduction of an Okinawan-based Nordic diet in health and in type 2 diabetes. A rapid weight reduction was observed after 2 weeks, which continued during the 12-week intervention and was mainly kept at follow-up. After 12 weeks, also plasma levels of lipids, CCK, GLP-1, glucagon, leptin, and PAI-1 were lowered. The lower levels of PYY persisted at follow-up, when also ghrelin and visfatin were lowered. Lower levels of plasma IL-18 after 12 weeks, and plasma haptoglobin and blood thrombocytes at follow-up, point to a decreased inflammation after introduction of the diet. Zonulin levels in serum was elevated during and after the dietary intervention. The metabolic changes could not be explained by any changes in gut microbiota diversity, amount of Enterobacteriaceae, or SCFAs levels.

The concentrations of CCK, GIP, GLP-1, and PYY are directly affected by the food composition in the gastrointestinal tract. Although the diet had a relative high fat and protein content, the secretion of CCK and PYY were reduced after the dietary intervention (33). However, the study participant's ordinary food may have had higher total amount of fat and protein than the Okinawan-based Nordic diet. The modified food consistency and composition of carbohydrates and fat may have led to the decreased concentrations of endogenous insulin, GIP, and GLP-1 observed (36-38). Correlations were found between concentrations of GIP and insulin during breakfast in health, and between GIP and C-peptide and insulin after the dietary intervention in type 2 diabetes. GIP have anabolic effects since it increases both the secretion and the effect of insulin on its receptor, thereby promoting accumulation of fat in adipose tissue (39). GIP is together with carbohydrates and hyperinsulinemia strongly involved in lipid regulation in man (10). The strong correlation between plasma levels of insulin and GIP and triglycerides in the breakfast study is in agreement with previous research $(10,40)$. Chronic hyperinsulinemia is considered to be the driving hormone in the metabolic syndrome as it disrupts metabolic balance and 
favors anabolic metabolism, which leads to increased body weight, carbohydrate craving, insulin resistance, and thereby further anabolic metabolism (41). Therefore, an overactive entero-insular axis is considered to be of importance in the pathogenesis of diabetes and obesity which demands altered chemical and physical food composition, as well as altered eating behaviors, to be reduced $(37,42)$. Weight reduction may thus be achieved both by a better diet composition and lower GIP and insulin levels.

Plasma triglycerides were equally increased after two different breakfast meals, despite the higher fat content in the control breakfast compared with the Okinawan-based Nordic breakfast (28). During the 12 weeks of dietary intervention, the triglyceride levels were lowered although a high fat energy percentage was ingested. These improvements may theoretically depend on the endocrine improvements regarding GIP and insulin secretions described above.

Both orexigenic (ghrelin) and anorexigenic hormone levels (CCK, glucagon, GLP-1, insulin, leptin, and PYY) were decreased in the present study. PYY remained lowered at follow-up, which is expected after weight reduction, in contrast to ghrelin, which is elevated after weight reduction $(16,43)$. Protein is the most satiating macronutrient, and protein quality and protein kinetics may be the most important factors in appetite regulation. Our findings of poor correlation between satiety and plasma levels of anorexigenic and orexigenic hormones are in line with the literature (44). After an acute weight loss, the body react with increased appetite to regain weight, reflecting a continuing role for the gut-brain axis in regulation of food intake and maintenance of body weight (16). It is therefore surprising that the degree of satiety remained the same or stronger during the dietary intervention (31), accompanied with decreased ghrelin levels at follow-up (33). Most hormones downregulate the ghrelin secretion (45), but since all other hormone levels measured in plasma also were lowered, this could not explain the low ghrelin levels. However, there seems to be nutrient-specific effects on ghrelin levels, and increased physical activity rendered lower basal plasma levels of ghrelin (16). The combination of protein-rich food and energy-restriction, which is the criteria of the Okinawan-based diet, is excellent to induce satiety and maintain weight-reduction (44). Our findings of decreased ghrelin levels may explain that the weight remained lowered at follow-up. A combination of physiological, behavioral, and environmental variables have been suggested to be measured in future studies to predict maintained weight loss (46).

The diabetes patients exhibited signs of inflammation at study start, as previously described in the literature (2), with elevated levels of hs-CRP and feces calprotectin, which were unaffected by the diet (34). Although a single meal did not affect the cytokine concentrations, the levels of IL-18 were lowered after 12 weeks of the dietary intervention in type 2 diabetes (35), accompanied with lower levels of haptoglobin and thrombocytes after 28 weeks (34). Both resistin and visfatin are pro-inflammatory factors which are proposed to promote atherosclerosis and general inflammation (47), and resistin and visfatin may be one of the best measurements to reflect an adaptation to a new metabolic balance and inflammatory steady state (33). Our findings were in line with previous research showing that weight reduction is followed by a greater downregulation of visfatin levels than of resistin levels (48), but we could not confirm the findings of correlation between resistin and insulin resistance and that visfatin has insulin-sensitizing effects leading to lower glucose levels (47). Most previous studies have described the correlations at one specific occasion. In this prospective interventional study, we could show how the reduced insulin resistance was seen already after 2 weeks (30), and thus preceded the reduced resistin and visfatin levels at the follow-up 26 weeks later. The inverse correlations between resistin and visfatin and carbohydrate and fiber intake are interesting. Our findings are in line with a recent meta-analysis on healthy Nordic diet, which showed that this diet decreased cardio-metabolic risk factors (49).

Zonulin is mainly secreted by the liver and is a biomarker of the metabolic syndrome, in agreement with positive correlations with weight, BMI, waist circumference, and levels of glucose, triglycerides, cholesterol, in both the present and former studies $(34,50)$. The suggestion that zonulin could be a biomarker of intestinal permeability has not been able to confirm in our experiments $(4,34,50)$. Furthermore, there seems to be a close relation between dietary factors and the levels of calprotectin and zonulin, with inverse correlation between zonulin and energy percentage of carbohydrates (34). The elevation of serum zonulin was unexpected since both weight reduction and improved metabolic parameters, which correlated with zonulin levels in diabetes (34) and in a healthy general population (50), should have reduced the zonulin secretion (2). The elevated zonulin levels may either be due to a direct effect of the dietary composition or altered secretion from the liver due to weight reduction. Since zonulin is the precursor of haptoglobin (51), which was lowered after 28 weeks, a third alternative is that the increased zonulin levels represent an arrest in the pathway to develop the fully expression of haptoglobin. However, recent research has shown that low carbohydrate content $(<50-55 \%)$ increases mortality compared to medium carbohydrate content usually present in Nordic diet $(26,49,52)$. The increased zonulin levels observed after this low carbohydrate diet may be a key point in this higher mortality risk, since zonulin is supposed to be a biomarker of unhealthy conditions $(4,50)$.

It remains to determine which of the ingredients in the food that are responsible for the observed hormonal and metabolic responses. The physical properties of the food were altered through higher fiber intake demanding more mastication, slower eating, greater gastric volume, and delayed gastric emptying rate (30). The chemical characteristics were changed with reduction of GI and processed food, lower energy intake of carbohydrates, and higher energy intake of fat and protein. In addition, most subjects changed food habits from irregular to regular meal habits (30). Regular consumption of breakfast cereals, especially whole grains, is associated with less overweight and development and management of diabetes (53). At follow-up after 28 weeks, the majority of the diabetes patients still prepared the breakfast in accordance to the recommendations. This was the dietary advice most easy to adhere to.

Thus, only adherence to the breakfast cereals and regular meals may be enough to reduce risk factors of metabolic diseases. In epidemiologic studies, mortality was lower in subjects with a high intake of whole-grain 
products; especially intake of breakfast cereals and non-white bread (54). However, the association between high intake of whole-grain products and overall healthier lifestyle habits, may reduce the association between whole grains and lower mortality to be markers of healthy habits $(55,56)$, instead of reflecting causality.

\section{Conclusion}

A single breakfast of the Okinawan-based Nordic diet with increased contents of fiber, fat, and protein, increases satiety and improves glucose homeostasis with lower levels of GIP and insulin in healthy volunteers. An intervention with this diet for 12 weeks in type 2 diabetes renders weight reduction with improved glucose and lipid metabolism. The satiety is increased and sweet cravings are decreased, which may explain the maintained weight reduction at follow-up, with lower levels of ghrelin, PYY, and visfatin compared to prior study start. The endocrine profile is in alignment with the improvements in anthropometric and metabolic homeostasis. At the same time, the levels of interleukin-18 are lowered, whereas levels of hs-CRP are unaffected, and zonulin levels are elevated. The mechanisms behind the metabolic improvements seem not to be mediated through alterations in gut microbiota. The total effect on health, morbidity, and mortality has to be further evaluated in long-term, controlled studies.

\section{Acknowledgements}

The author would like to acknowledge the staff Igelösa Life Science AB, Lund, who supplied and prepared the meals and the staff at the Internal Medicine Research Group, Skåne University Hospital, Malmö, for performing the trials.

\section{Funding}

These studies were supported by grants from King Gustaf V's and Queen Victoria Free Maison's Foundation (grant no. 20141204), Dir Albert Påhlsson's Foundation (FB2014-0001), Development Foundation of Region Skåne (grant no. 2014/354), and Foundation of Skåne University Hospital (grant no. 1500291).

\section{Availability of data and materials}

The datasets used and/or analyzed during the current study are available from the corresponding author on reasonable request.

\section{Authors' contributions}

$\mathrm{BO}$ was the principal investigator for the clinical trials, wrote the manuscript and agreed to be accountable for all aspects of the work in ensuring that questions related to the accuracy or integrity of any part of the work are appropriately investigated and resolved.

\section{Ethics approval and consent to participate}

The subjects were treated according to the Declaration of Helsinki and the studies were approved by the Regional Ethics
Review Board at Lund University (Project identification code: 2014/460; Date of approval: 4 September 2014). All subjects gave their written, informed consent before entering the study, which was monitored by an external monitor and registered at ClinicalTrials.gov data base (NCT02405806).

\section{Patient consent for publication}

All subjects provided their written, informed consent for publication.

\section{Competing interests}

The author declares that he has no competing interest.

\section{References}

1. International diabetes federation. IDF diabetes atlas, 7 edition. Brussels, Belgium: International Diabetes Federation, 2015.

2. Calder PC, Ahluwalia N, Brouns F, Buetler T, Clement K, Cunningham K, Esposito K, Jönsson LS, Kolb H, Lansink M, et al: Dietary factors and low-grade inflammation in relation to overweight and obesity. Br J Nutr 106 (Suppl 3): S5-S78, 2011.

3. de Punder K and Pruimboom L: The dietary intake of wheat and other cereal grains and their role in inflammation. Nutrients 5: 771-787, 2013.

4. Fasano A: Intestinal permeability and its regulation by zonulin: Diagnostic and therapeutic implications. Clin Gastroenterol Hepatol 10: 1096-1100, 2012.

5. Qi L and Hu FB: Dietary glycemic load, whole grains, and systemic inflammation in diabetes: The epidemiological evidence. Curr Opin Lipidol 18: 3-8, 2007.

6. Casas R, Sacanella E and Estruch R: The immune protective effect of the Mediterranean diet against chronic low-grade inflammatory diseases. Endocr Metab Immune Disord Drug Targets 14: 245-254, 2014.

7. Schwab U, Lauritzen L, Tholstrup T, Haldorssoni T, Riserus U, Uusitupa M and Becker W: Effect of the amount and type of dietary fat on cardiometabolic risk factors and risk of developing type 2 diabetes, cardiovascular diseases, and cancer: A systematic review. Food Nutr Res 58, 2014.

8. AL-Suhaimi EA and Shehzad A: Leptin, resistin and visfatin: The missing link between endocrine metabolic disorders and immunity. Eur J Med Res 18: 12, 2013.

9. Tilg $\mathrm{H}$ and Moschen AR: Role of adiponectin and PBEF/visfatin as regulators of inflammation: Involvement in obesity-associated diseases. Clin Sci (Lond) 114: 275-288, 2008.

10. Nordt TK, Sawa H, Fujii S, Bode C and Sobel BE: Augmentation of arterial endothelial cell expression of the plasminogen activator inhibitor type-1 (PAI-1) gene by proinsulin and insulin in vivo. J Mol Cell Cardiol 30: 1535-1543, 1998.

11. Anagnostis P, Athyros VG, Tziomalos K, Karagiannis A and Mikhailidis DP: Clinical review: The pathogenetic role of cortisol in the metabolic syndrome: A hypothesis. J Clin Endocrinol Metab 94: 2692-2701, 2009.

12. Yoder SM, Yang Q, Kindel TL and Tso P: Stimulation of incretin secretion by dietary lipid: Is it dose dependent? Am J Physiol Gastrointest Liver Physiol 297: G299-G305, 2009.

13. Yoder SM, Yang Q, Kindel TL and Tso P: Differential responses of the incretin hormones GIP and GLP-1 to increasing doses of dietary carbohydrate but not dietary protein in lean rats. Am J Physiol Gastrointest Liver Physiol 299: G476-G485, 2010.

14. Asmar M, Simonsen L, Madsbad S, Stallknecht B, Holst JJ and Bülow J: Glucose-dependent insulinotropic polypeptide may enhance fatty acid re-esterification in subcutaneous abdominal adipose tissue in lean humans. Diabetes 59: 2160-2163, 2010.

15. Holst JJ, Vilsbøll T and Deacon CF: The incretin system and its role in type 2 diabetes mellitus. Mol Cell Endocrinol 297: $127-136,2009$.

16. Gutierrez-Aguilar R and Woods SC: Nutrition and L and K-enteroendocrine cells. Curr Opin Endocrinol Diabetes Obes 18: 35-41, 2011.

17. Overduin J, Gibbs J, Cummings DE and Reeve JR Jr: CCK-58 elicits both satiety and satiation in rats while CCK-8 elicits only satiation. Peptides 54: 71-80, 2014. 
18. Cooper JA: Factors affecting circulating levels of peptide YY in humans: A comprehensive review. Nutr Res Rev 27: 186-197, 2014.

19. Chu S and Schubert ML: Gastric secretion. Curr Opin Gastroenterol 29: 636-641, 2013

20. Lean ME and Malkova D: Altered gut and adipose tissue hormones in overweight and obese individuals: Cause or consequence? Int J Obes (Lond) 40: 622-632, 2016.

21. He X, Marco ML and Slupsky CM: Emerging aspects of food and nutrition on gut microbiota. J Agric Food Chem 61: 9559-9574, 2013.

22. Wagner RD: Effects of microbiota on GI health: Gnotobiotic research. Adv Exp Med Biol 635: 41-56, 2008

23. Gjymishka A, Coman RM, Brusko TM and Glover SC: Influence of host immunoregulatory genes, ER stress and gut microbiota on the shared pathogenesis of inflammatory bowel disease and Type 1 diabetes. Immunotherapy 5: 1357-1366, 2013.

24. Karlsson F, Tremaroli V, Nielsen J and Bäckhed F: Assessing the human gut microbiota in metabolic diseases. Diabetes 62: 3341-3349, 2013.

25. Willcox DC, Scapagnini G and Willcox BJ: Healthy aging diets other than the Mediterranean: A focus on the Okinawan diet. Mech Ageing Dev 136-137: 148-162, 2014.

26. Nordic Council of Ministers: Nordic Nutrition Recommendations 2012. https://norden.diva-portal.org/smash/get/diva2:704251/ FULLTEXT01.pdf

27. Ohlsson B, Höglund P, Roth B and Darwiche G: Modification of a traditional breakfast leads to increased satiety along with attenuated plasma increments of glucose, C-peptide, insulin and glucose-dependent insulinotropic polypeptide in humans. Nutr Res 36: 359-368, 2016.

28. Ohlsson B, Darwiche G, Roth B and Höglund P: Two meals with different carbohydrate, fat and protein contents render equivalent postprandial plasma levels of calprotectin, cortisol, triglycerides and zonulin. Int J Food Sci Nutr 67: 872-880, 2016.

29. Huang F, Nilholm C, Roth B, Linninge C, Höglund P, Nyman M and Ohlsson B: Anthropometric and metabolic improvements in human type 2 diabetes after introduction of an Okinawan-based Nordic diet are not associated with changes in microbial diversity or SCFA concentrations. Int J Food Sci Nutr 69: 729-740, 2018.

30. Darwiche G, Höglund P, Roth B, Sjöberg T, Wohlfart B, Steen S and Ohlsson B: An Okinawan-based Nordic diet improves anthropometry, metabolic control, and health-related quality of life in Scandinavian patients with type 2 diabetes: A pilot trial. Food Nutr Res 60: 32594, 2016.

31. Ohlsson B, Darwiche G, Roth B, Bengtsson M and Höglund P: High fiber fat and protein contents lead to increased satiety reduced sweet cravings and decreased gastrointestinal symptoms independently of anthropometric hormonal and metabolic factors. J Diabet Metabol 8: 733, 2017

32. Todd DTU: Endocrinology and metabolism 2016 HOMA2 calculator. Oxford, UK: The University of Oxford, 2016.

33. Ohlsson B, Darwiche G, Roth B and Höglund P: Alignments of endocrine, anthropometric, and metabolic parameters in type 2 diabetes after intervention with an Okinawan-based Nordic diet. Food Nutr Res 14: 62, 2018

34. Ohlsson B, Roth B, Larsson E and Höglund P: Calprotectin in serum and zonulin in serum and feces are elevated after introduction of a diet with lower carbohydrate content and higher, fat and protein contents. Biomed Rep 6: 411-422, 2017.

35. Nilholm C, Roth B, Höglund P, Blennow K, Englund E, Hansson O, Zetterberg $\mathrm{H}$ and Ohlsson B: Dietary intervention with an Okinawan-based Nordic diet in type 2 diabetes renders decreased interleukin-18 concentrations and increased neurofilament light concentrations in plasma. Nutr Res 60: 13-25, 2018.

36. Shimotoyodome A, Fukuoka D, Suzuki J, Fujii Y, Mizuno T, Meguro S, Tokimitsu I and Hase T: Coingestion of acylglycerols differentially affects glucose-induced insulin secretion via glucose-dependent insulinotropic polypeptide in C57BL/6J mice. Endocrinology 150: 2118-2126, 2009.

37. Peracchi M, Santangelo A, Conte D, Fraquelli M, Tagliabue R, Gebbia $\mathrm{C}$ and Porrini M: The physical state of a meal affects hormone release and postprandial thermogenesis. Br J Nutr 83: $623-628,2000$
38. Smeets AJ, Soenen S, Luscombe-Marsh ND, Ueland $\varnothing$ and Westerterp-Plantenga MS: Energy expenditure, satiety, and plasma ghrelin, glucagon-like peptide 1, and peptide tyrosine-tyrosine concentrations following a single high-protein lunch. J Nutr 138: 698-702, 2008.

39. Heller RF and Heller RF: Hyperinsulinemic obesity and carbohydrate addiction: The missing link is the carbohydrate frequency factor. Med Hypotheses 42: 307-312, 1994.

40. Runchey SS, Valsta LM, Schwarz Y, Wang C, Song X, Lampe JW and Neuhouser ML: Effect of low- and high-glycemic load on circulating incretins in a randomized clinical trial. Metabolism 62: 188-195, 2013.

41. Kopp W: High-insulinogenic nutrition-an etiologic factor for obesity and the metabolic syndrome? Metabolism 52: 840-844, 2003.

42. Yip RG and Wolfe MM: GIP biology and fat metabolism. Life Sci 66: 91-103, 2000.

43. Sumithran P, Prendergast LA, Delbridge E, Purcell K, Shulkes A, Kriketos A and Proietto J: Long-term persistence of hormonal adaptations to weight loss. N Engl J Med 365: 1597-1604, 2011.

44. Bendtsen LQ, Lorenzen JK, Bendsen NT, Rasmussen C and Astrup A: Effect of dairy proteins on appetite, energy expenditure, body weight, and composition: A review of the evidence from controlled clinical trials. Adv Nutr 4: 418-438, 2013.

45. Iwakura $\mathrm{H}$, Kangawa $\mathrm{K}$ and Nakao $\mathrm{K}$ : The regulation of circulating ghrelin-with recent updates from cell-based assays. Endocr J 62: 107-122, 2015.

46. Strohacker K, McCaffery JM, MacLean PS and Wing RR: Adaptations of leptin, ghrelin or insulin during weight loss as predictors of weight regain: A review of current literature. Int J Obes (Lond) 38: 388-396, 2014.

47. Stofkova A: Resistin and visfatin: Regulators of insulin sensitivity, inflammation and immunity. Endocr Regul 44: 25-36, 2010.

48. Sheu WH, Chang TM, Lee WJ, Ou HC, Wu CM, Tseng LN, Lang HF, Wu CS, Wan CJ and Lee IT: Effect of weight loss on proinflammatory state of mononuclear cells in obese women. Obesity (Silver Spring) 16: 1033-1038, 2008.

49. Ramezani-Jolfaie N, Mohammadi M and Salehi-Abargouei A: The effect of healthy Nordic diet on cardio-metabolic markers: A systematic review and meta-analysis of randomized controlled clinical trials. Eur J Nutr: Aug 20, 2018 (Epub ahead of print).

50. Ohlsson B, Orho-Melander M and Nilsson PM: Higher levels of serum zonulin may rather be associated with increased risk of obesity and hyperlipidemia, than with gastrointestinal symptoms or disease manifestation. Int J Mol Sci 18: 582, 2017.

51. Vanuytsel T, Vermeire S and Cleynen I: The role of haptoglobin and its related protein, zonulin, in inflammatory bowel disease. Tissue Barriers 1: e27321, 2013.

52. Seidelmann SB, Claggett B, Cheng S, Henglin M, Shah A, Steffen LM, Folsom AR, Rimm EB, Willett WC and Solomon SD: Dietary carbohydrate intake and mortality: A prospective cohort study and meta-analysis. Lancet Public Health 3: e419-e428, 2018.

53. Carlson O, Martin B, Stote KS, Golden E, Maudsley S, Najjar SS, Ferrucci L, Ingram DK, Longo DL, Rumpler WV, et al: Impact of reduced meal frequency without caloric restriction on glucose regulation in healthy, normal-weight middle-aged men and women. Metabolism 56: 1729-1734, 2007.

54. Williams PG: The benefits of breakfast cereal consumption: A systematic review of the evidence base. Adv Nutr 5: 636S-673S, 2014.

55. Johnsen NF, Frederiksen K, Christensen J, Skeie G, Lund E, Landberg R, Johansson I, Nilsson LM, Halkjær J, Olsen A, et al: Whole-grain products and whole-grain types are associated with lower all-cause and cause-specific mortality in the Scandinavian HELGA cohort. Br J Nutr 114: 608-623, 2015.

56. Egeberg R, Frederiksen K, Olsen A, Johnsen NF, Loft S, Overvad $\mathrm{K}$ and Tjønneland A: Intake of wholegrain products is associated with dietary, lifestyle, anthropometric and socio-economic factors in Denmark. Public Health Nutr 12: $1519-1530,2009$ 